\title{
A Biomechanical Analysis on the Sensitivity of Bone Graft and Osteotomy Orientation in Relation to Post-Operative Stability in Open Wedge High Tibial Osteotomy*
}

\author{
Hyun KIM**, Kyung Wook NHA*** and Sung Jae LEE** \\ ** Department of Biomedical Engineering, Inje University, \\ 607 Obang-dong, Gimhae-si, Gyeongsangnam-do 621-749, Korea \\ *** Department of Orthopedic Surgery, Ilsanpaik Hospital, \\ Daehwa-dong, Ilsanseo-gu, Goyang-si, Gyeonggi-do, Korea \\ E-mail: sjl@bme.inje.ac.kr
}

\begin{abstract}
Potential complications after open wedge high tibial osteotomy (HTO) still remain unanswered as they are known to be primarily dependent on the surgical technique or the fixation strength. In this study, we evaluated the sensitivity of surgical variations (presence of bone graft, osteotomy line orientation and loading pattern) affecting post-operative stability using finite element analysis. Changes in stress distribution were also assessed at the lateral cortex bone and bone plate. A total six types of post-operative FE model were constructed to accommodate surgical variations based on the validated intact tibia model; Case 1 (lower level osteotomy, with no bone graft or with auto-tri-cortical bone), Case 2 (safe level osteotomy, with no bone graft or with auto-tri-cortical bone), Case 3 (upper level osteotomy, with no bone graft or with auto-tri-cortical bone). Two types of loading condition (axial compression_2450N with bending_240N and torsion_15Nm) were imparted. The use of bone graft material at the osteotomy site decreased the stress distribution at the lateral cortex bone and bone screw. And the lower level provided more post-operative stability than other osteotomy level (safe, upper). However, the 'safe' zone offered relatively similar results to those of the 'lower' zone. The osteotomy line near the lower end of the 'safe' zone was indeed the safest and most practical surgical approach as suggested in previous clinical studies. Therefore, our results suggested the use of bone graft with safe level osteotomy to assure the greatest post-operative stability and to reduce the likelihood of correction loss.
\end{abstract}

Key words: Open Wedge High Tibia Osteotomy, Finite Element Analysis, Surgical Variations, Bone Graft, Osteotomy Level

\section{Introduction}

High tibial osteotomy (HTO) is gaining recognition as an established surgical technique for the treatment of medial unicompartmental osteoarthritis and osteonecrosis of the knee. Studies have shown that HTO can be effective in delaying the degenerative process and relieving knee pain especially for young and elderly patients who have physically active life styles ${ }^{(1,2)}$. Specific aims of HTO are to realign the mechanical axis of the knee and to redistribute weight-bearing stresses from the degenerated medial compartment of knee to the lateral part that is more intact and relatively less degenerated ${ }^{(2,3)}$. Depending on the location and the extent of the osteotomy involved, two operative methods for HTO are

${ }^{*}$ Received 28 May, 2012 (No. 12-0232) [DOI: 10.1299/jbse.7.358]

Copyright $\odot 2012$ by JSME 
known: one is the conventional lateral closed-wedge HTO and the other medial open-wedge $\mathrm{HTO}^{(4)}$. The surgical procedures for the lateral closed-wedge HTO include performing the osteotomy from the lateral side along with the fibula to the medial side and removing the bone wedge at the lateral proximal tibia with a dissection of the lateral soft tissue by ${ }^{(5,6)}$. The reported advantages of this technique are better initial stability with rapid healing and no need for bone grafting or graft substitute. However, complications from the fibular osteotomy or neurovascular injury on the proximal tibiofibular joint have been reported ${ }^{(5,6)}$. In recent years, the medial open-wedge HTO have been introduced to overcome aforementioned shortfalls. Recent studies suggest that no alteration of the fibular nerve injury, no disruption of the proximal tibiofibular joint, and preservation of the osseous substance from potential total knee arthroplasty can be possible ${ }^{(2,3,7,8)}$. In fact, favorable clinical results have been reported with more accurate correction of varus deformity compared to a conventional lateral closed-wedge HTO ${ }^{(9,10)}$. However, potential complications such as collapse of the lateral cortex and implant fracture with accompanying loss of correction still remain unanswered as they are known to be primarily dependent on bone quality of patients, the surgical technique itself, or the strengths of bone graft and fixation plate ${ }^{(11,12)}$.

In vitro biomechanical studies have been performed to evaluate the structural stability and rigidity of the post-operative knee with various types of plates and bone graft with HTO $(13,14)$. They showed that the plate design greatly influences the structural stability after open wedge HTO. Another study evaluated the structural rigidity and fixation strength between only plate fixation and plate fixation with bone graft ${ }^{(15)}$ and found that the use of plate with bone graft is likely to improve the stability at the osteotomy site compared with no bone graft. For more parametrical analyses on the surgical and structural factors involved, finite element (FE) studies have been done to assess the postoperative stability. They include studies on biomechanical effects in relation to plate location of plate, changes in stress distribution of the tibia bony structure, and structural rigidity of the construct ${ }^{(16)}$. However, effects of many surgical factors on the structural integrity after surgery still remain unknown. Particularly, the optimal extent of the osteotomy and resulting amount of the bone graft in relation to the surgical techniques and pre-operative diagnosis of the patient need to be elucidated to reduce the likelihood of complications from structural failure $^{(3,17)}$.

In this study, we constructed a 3-D post-operative tibia FE model to quantitatively investigate the sensitivity of various factors (presence of bone graft, osteotomy line orientation and loading pattern) affecting structural stability after open wedge HTO. Changes in stress distribution at the lateral cortex bone and bone plate were also assessed.

\section{Materials and Methods}

\subsection{Construction of the post-operative model}

The geometry of the three-dimensional (3-D) intact tibia finite element (FE) model was constructed as follows. First, the geometry of the intact tibia model (right side) was obtained based on high resolution computed tomography (CT) scans (at 1-mm in thickness) of a 27 -year-old man (height, $170 \mathrm{~cm}$; weight, $70 \mathrm{~kg}$ ) with no pathologies (Fig. 1 (a)). The inner and outer contours of the surface of the tibia were extracted from computed tomograms (CT) with the method of thresholding and subsequent 3-D volume was reconstructed by stacking the extracted contours (Fig. 1 (b)). The 3-D geometry model was subdivided with hexahedron solids in uniform meshing (Fig. 1 (c) and 1 (d)). Finally, 3-D isotropic eight-noded solid elements (C3D8 element type in general FE package program, ABAQUS, Version 5.6, Hibbit, Karlson, Sorenson Inc, Pawtucket, RI, USA) was used for modeling the cortical and cancellous bones (Fig. 1 (f)). This intact tibia FE model consisted 
of 54477 nodes and 48451 elements and represent pre-operative stage. FE Post-processing was done with ABAQUS. The material properties of each element (cortical bone, $\mathrm{E}=17000 \mathrm{MPa}, \mathrm{v}=0.32$; cancellous bone, $\mathrm{E}=104 \mathrm{MPa}, \mathrm{v}=0.32$ ) were taken from the literature $(18,19)$, and the bone was assumed to be linearly elastic and isotropic. This intact tibia model was validated by comparing the strain results to the previous biomechanical test data ${ }^{(20)}$ under same axial compressive load condition $(2030 \mathrm{~N})$ at 10 different locations (3 points each at the anteromedial and anterolateral regions, 4 points at the posterior in Fig. 2 (a)). Relative differences between our FE results and the published values were within $7 \%$ at all locations (Fig. 2 (b)).

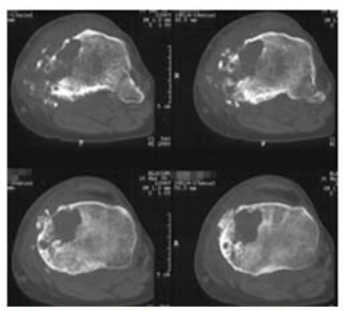

(a) Geometry data extraction from $\mathrm{CT}$ images

$<$ Mimics 13.1, Materialise Inc., Louvain, Belgium>

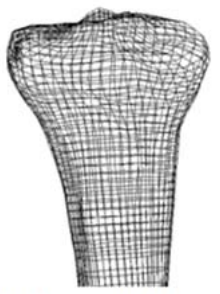

(c) Surface model segmentation $<$ Rhinoceros 3.0, Robert McNeel \& Associates Corp., WA, USA>

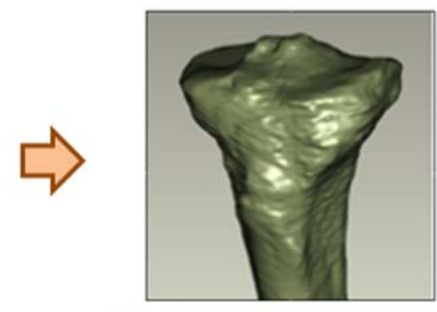

(b) 3-D geometry reconstruction $<$ Mimics 13.1, Materialise Inc. Louvain, Beligum>

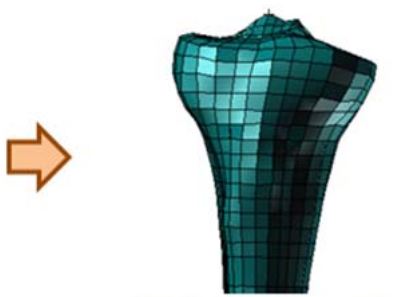

(d) Solid model generation $<$ Patran 2006, MSC. Software Corp., CA, USA>

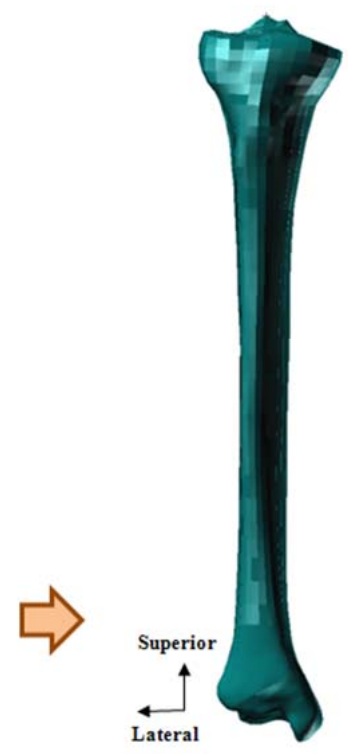

(f) 3-D FE model

Figure 1. Construction of a 3-D FE model of the intact (pre-operative) tibia from the CT-scans of a normal person (Software used during each step is included)

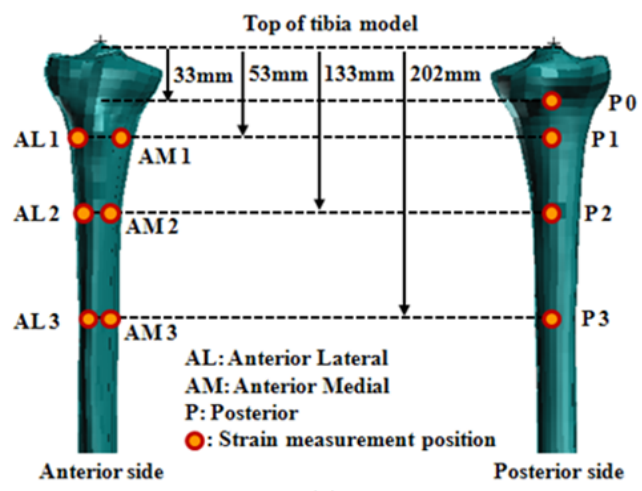

(a)

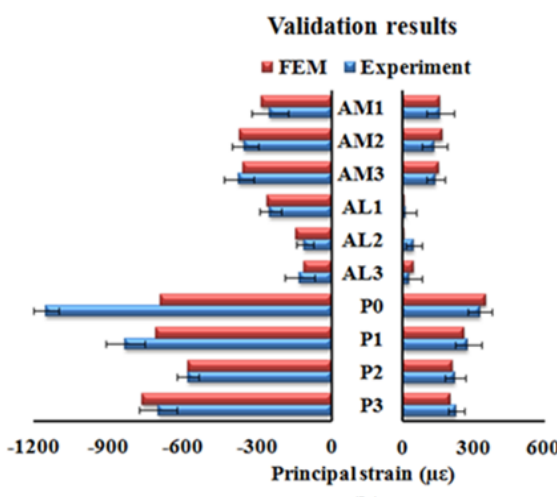

(b)

Figure 2. Validation of FE model by comparing our FE strain results (FEM) with the experimental results (Experiment) from literature (ref. 20) under identical loading conditions; (a) measurement locations, (b) strain results

\subsection{Clinical factors considered}

The surgical technique of open-wedge HTO underwent various in the fixation technique 
and augmentation with bone graft.

First, the incidence of lateral cortex failure ranges about $12 \sim 18 \%$ based on clinical follow-up studies $(11,12)$. The likelihood of fracture increases if the end point of the osteotomy at the lateral side is too close to the tibial plateau ${ }^{(21)}$ and the lateral cortex hinge should be retained for added stability during gap distraction following osteotomy ${ }^{(17)}$. A clinical finding suggests that osteotomy should be performed along this laterally-inclined plane within the so-called "safe zone" to ensure minimal risk of fracture ${ }^{(22)}$. In a previous study, the "safe zone" was defined as the ideal end position of the plane of the osteotomy near the tibiofibular joint. It was suggested that the plane be oriented obliquely to end between the tip and the circumference line of the fibular head (Fig. 3). Generally in this case, the end point of osteotomy would be located at about $10 \sim 15 \mathrm{~mm}$ below the tibial plateau as compared to the starting point of about $45 \mathrm{~mm}$. It is assumed that this would protect the tibiofibular joint and surrounding soft tissues which would provide added support and significantly reduce the risk of lateral cortex fracture as compared to an osteotomy directed at a more distal plane (distal to the circumference line of the fibular head). Despite clinical findings and suggestions, sensitivity of the extent of the osteotomy remain unknown especially in relation to post-operative structural stability Thus, we investigated the biomechanical effects of osteotomy level change in relation to stability of lateral cortex hinge by assessing the stress distribution. Three types of osteotomy level were chosen based on the distance below the tibia plateau line (Fig. 4 (a)); upper level (5mm, the most proximal to the tibial plateau), safe level (10mm, suggested as the "safe zone"), lower level $(20 \mathrm{~mm}$, the most distal to the tibial plateau). These distances were selected based on the clinical experience of one of the authors.

Second, open wedge HTO requires the bone graft to reinforce the bone loss volume left void following corrective procedure after osteotomy ${ }^{(15,23)}$. Autogenous iliac graft has been used routinely as a part of the technique ${ }^{(23)}$. However, the surgical time and possible morbidity including chronic pain, infection, palpable defects, paresthesia and increased blood loss are drawbacks of the procedures ${ }^{(24,25)}$. So, the question of a satisfactory bone union after HTO without bone graft has been raised. In this study, we constructed the two types of bone graft model to verify the effect of bone graft insertion on post-operative stability (Fig. 4 (b)); no bone graft, auto-tri-cortical bone $(E=17000 \mathrm{MPa}, \mathrm{v}=0.32)$. The dimension of the bone graft wedge was $10 \mathrm{~mm}(\mathrm{~W}) \times 32 \mathrm{~mm}$ (L) $\times 50 \mathrm{~mm}$ (D) based on the clinical experience of one of the authors in this study ${ }^{(23)}$.

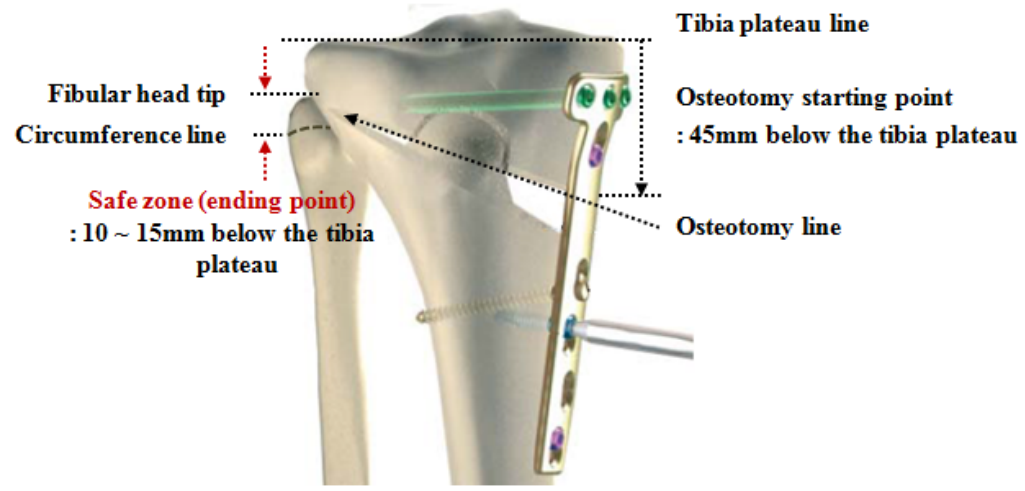

Figure 3. Definition of safe zone; osteotomy oriented to end between the tip of the fibular head and the circumference line of the fibular head.

\subsection{Construction of the post-operative model}

The post-operative model was made based on the standard technique recommended in literature (Fig. 3) ${ }^{(3,7,22)}$. The standardized osteotomy was performed by starting from the 
medial cortex $40 \mathrm{~mm}$ below the medial tibia plateau line to the end point near the lateral side along the three different laterally-oblique lines (upper, safe, \& lower) depending on the end position of the osteotomy. The correction height on the medial cortex was set at $10 \mathrm{~mm}$ in all post-operative models based on previous studies ${ }^{(13,14)}$. Here, the space created by the osteotomy and distraction was filled either left void or with auto-tri-cortical bone graft, and subsequently was secured with TomoFix ${ }^{\mathrm{TM}}$ plate (pure titanium, $\mathrm{E}=105 \mathrm{GPa}, \mathrm{v}=0.3$, yield strength $=480 \mathrm{MPa}$ ). A total six types of post-operative FE model were constructed to accommodate surgical variations. The locking compression plate (LCP) was attached to the medial side of the tibia. The bone-screws interface behavior was accomplished via "tie" contact condition to assume complete postoperative bony union (26). And fully locking condition was assumed at the plate-screw interface whereas some movements were allowed between the plate and surrounding bone (frictional coefficient, $\mu=0.5$ ) ${ }^{(27)}$.

(a) Osteotomy level (below the tibia plateau line)

- Upper (5mm)

- Safe (10mm)

- Lower (20mm)

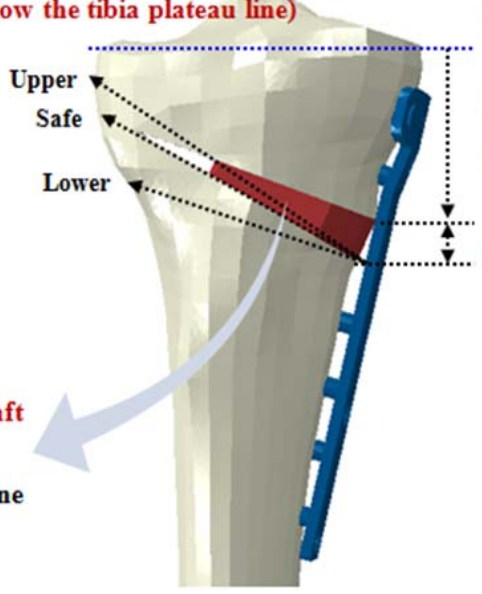

Tibia plateau line

Osteotomy starting point

: $45 \mathrm{~mm}$ below the tibia plateau line

(b) Presence of bone graft

- No bone graft

-Auto-tri-cortical bone

Correction height $(10 \mathrm{~mm})$

Figure 4. Surgical variations to construct the post-operative models; (a) osteotomy level, (b) presence of bone graft (No bone graft, immediately after surgery \& no bone union; auto-tri-cortcial bone, fully bony union \& complete bony healing)

\subsection{Loading and boundary conditions}

Physiologically-relevant loads were applied to the top of the tibia based on the body weight $(700 \mathrm{~N})$ of the subject before a toe-off stage (highest knee joint contact loading in gait cycles; $3.5 \mathrm{x}$ body weight) ${ }^{(28)}$. The distal ends of each post-operative model were completely fixed in all directions. Two types of loading condition (axial compression with bending and torsion) were imparted (Fig. 5). First, the compressive-bending load (axial compressive load, $2450 \mathrm{~N}$; bending load. $240 \mathrm{~N}$ ) was applied to the two nodal points corresponding to the centers of each tibial condyle (Fig. 5 (a)) as the mechanical axes of low limbs (Mikulicx-line) passes through $4 \pm 2 \mathrm{~mm}$ medial inside points from the center of knee joint ${ }^{(29)}$. Thus, the compression load was divided into $60 \%(1470 \mathrm{~N})$ and $40 \%(980 \mathrm{~N})$ to the medial and lateral sides, respectively ${ }^{(30,31)}$. The bending load was equally divided between both the medial and the lateral sides (120 N each). The second set of loading condition included torsional moment of $15 \mathrm{Nm}$ at the superior aspects of the tibial condyle (Fig. 5 (b) ${ }^{(30,31)}$. Most common post-op failures following open wedge HTO are either lateral cortex fractures or screw breakages. Thus, the fracture risk (\%) of each component (lateral cortex hinge_cortical bone $\&$ bone screw_pure titanium) was evaluated by the ratio between the peak von Mises stresses and the fatigue strength (cortical bone: $75 \mathrm{MPa} \_10^{7}$ cycles_compression \& 48MPa_15000 cycles_torsion; pure titanium: $290 \mathrm{MPa} \_10^{6}$ cycles) of each material $(32,33,34)$. The overall post-operative stability was assessed in terms of the 
structural rigidity as the applied load per displacement $(\mathrm{N} / \mathrm{mm})$ or rotation $\left(\mathrm{Nm} /{ }^{\circ}\right)$.

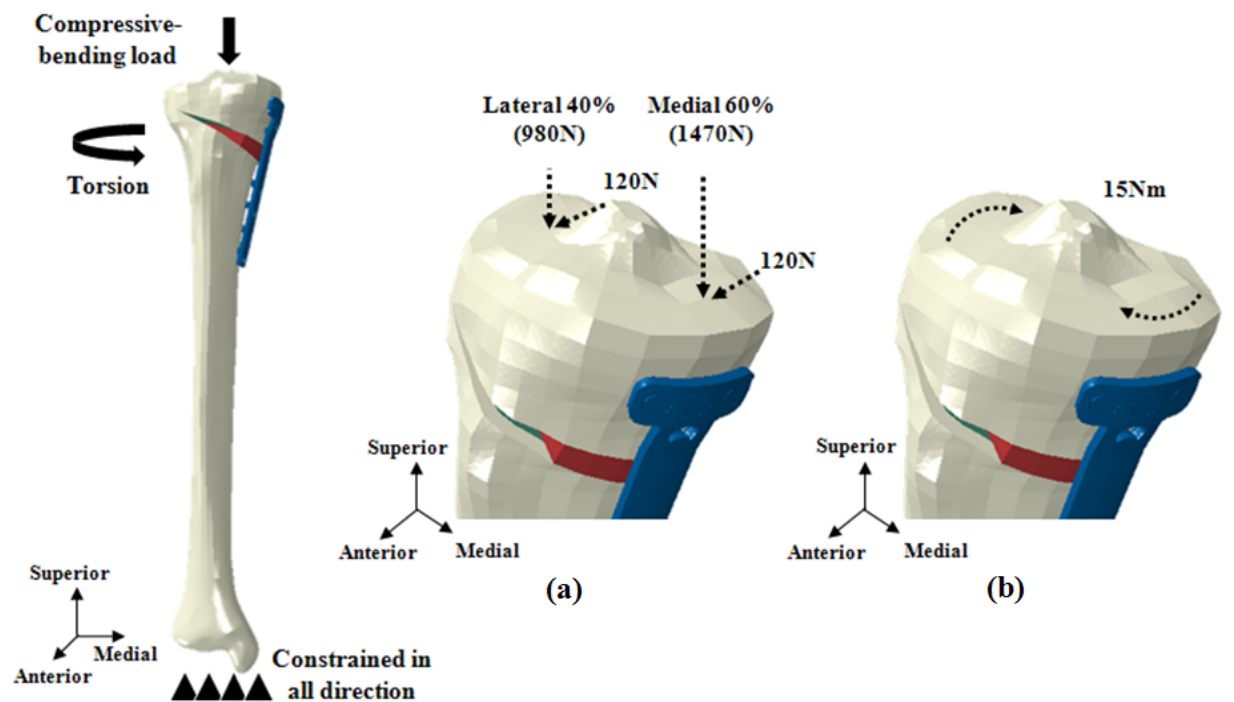

Figure 5. Loading and boundary conditions: (a) compressive-bending load, (b) torsional load.

\section{Results}

\subsection{Fracture risk of the lateral cortex bone}

Our results showed that the fracture risk of the lateral cortex bone was more sensitive to the bone graft insertion than the changes in osteotomy level (Fig. 6). With bone graft, the fracture risk was reduced by half in all cases. This was more evident as the osteotomy level moved more superiorly close to the tibial plateau as shown with the 'upper' placement. Compressive-bending load $(35 \sim 70 \%)$ consistently yielded higher fracture risk than the torsional load $(10 \sim 35 \%)$ regardless of surgical variations. The location of the highest stress was at the bone near the neighborhood in the lateral osteotomy site regardless of the loading conditions and affecting factors.
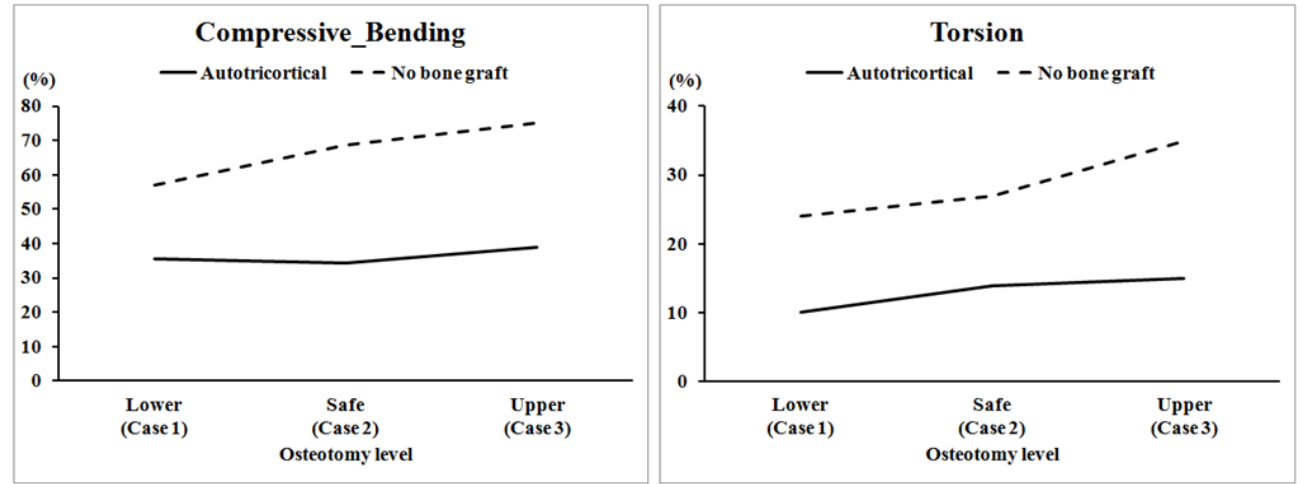

Figure 6. Changes in fracture risk of the lateral cortex of the tibia (\%, peak Von Mises stress / fatigue strength of material x 100)

\subsection{Fracture risk of the bone screw}

Fracture risk of the bone screw (Titanium locking head screws, $\phi=5.0 \mathrm{~mm}$, length $=60 \mathrm{~mm}$, pure titanium, $\mathrm{E}=105 \mathrm{GPa}, \mathrm{v}=0.3$ ) was reduced most significantly with bone graft against torsion from $35 \%$ to about $5 \%$. In other cases, it showed a more gradual 
decrease with overall ratio consistently lower than those of the lateral cortex bone (Fig. 7). Changes in the osteotomy level did not significantly affect the overall results; only slight decrease in risk was observed with the upper placement especially when no bone graft was inserted. Highest bone screw stresses were found at the 4th screw (during compression) and 5 th screw (during torsion).
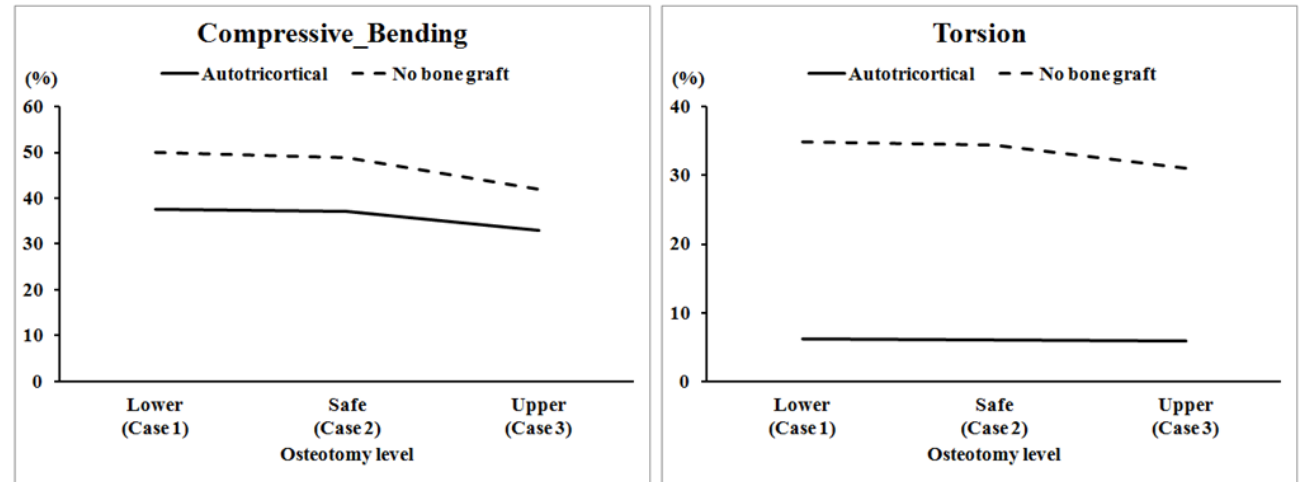

Figure 7. Changes in fracture risk of the bone screw (\%, peak Von Mises stress / fatigue strength of material x 100)

\subsection{Structural rigidity}

Overall post-operative structural rigidity was highest with the 'lower' osteotomy, followed by 'safe' and 'upper', regardless of the loading or the bone graft used (Fig. 8). This trend was more evident with the bone graft than without.
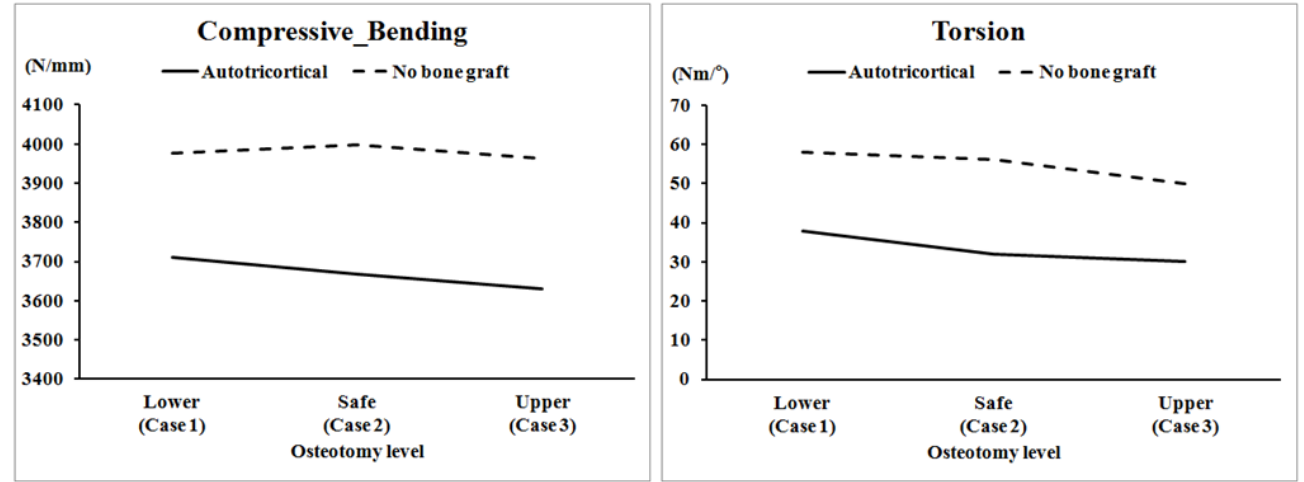

Figure 8. Changes in Structural rigidity of post-operative FE model

\section{Discussion}

Open wedge HTO has become an effective treatment option for the varus degenerative knee in young and active patient ${ }^{(1,2)}$ as it offers several advantages over the closed wedge HTO. Most significantly, precise correction of the deformity and conservation of bone stock for potential total knee arthroplasty are known to be some of the advantages ${ }^{(3,7,8)}$. But, the procedure is technically demanding and high failure rate (up to $45 \%$ ) has been reported. Collapse of lateral cortex and implant fracture with accompanying loss of correction ${ }^{(11)}$ are primarily dependent on surgical variations associated with the surgical technique or the strength of bone graft and fixation plate ${ }^{(11,12)}$. In this biomechanical comparative analysis study, the sensitivity of surgical factors such as the osteotomy line location and bone graft was investigated in relation to overall post-operative stability to suggest more optimal surgical procedures. 
The Puddu ${ }^{\mathrm{TM}}$ plate (Arthrex Inc., Naples, Florida, USA) and the TomoFix ${ }^{\mathrm{TM}}$ plate (Synthes Inc., Solothum, Switzerland) are two of the most popular bone plates currently used in open wedge HTO. The Puddu ${ }^{\mathrm{TM}}$ plate is a short plate with spacer block for prop the distracted osteotomy site. On the other hand, the TomoFix ${ }^{\mathrm{TM}}$ plate is a long plate with locking compression plate technique to preserve the bone blood supply, and this technique relies on locked screw heads with all six degrees of freedom restrained. In vitro biomechanical studies have been performed to evaluation primary stability of various different fixation plates ${ }^{(13,14)}$. The constructs with long plates resisted more than twice the amount of loading cycles compared to the short plates. They suggest that in patients with failed lateral cortex, the short plate design provides insufficient stability for bone healing and maintenance of the correction. Another studies also reported that the TomoFix ${ }^{\mathrm{TM}}$ plate provides primary stability in both compressive and torsional load compared with the Puddu $^{\mathrm{TM}}$ plate ${ }^{(35)}$. These results demonstrated that the TomoFix ${ }^{\mathrm{TM}}$ plate was provided the higher stability than Puddu ${ }^{\mathrm{TM}}$ plate regardless of loading conditions. Recently, many follow up studies recommended the surgical technique with TomoFix $^{\mathrm{TM}}$ plate to provide a successful clinical results based on the good results in $70 \sim 90 \%$ of cases ${ }^{(22,36,37)}$. Thus, we considered the long bone plate with eight screw holes in post operative model.

Another factor for failure or success of the open wedge HTO is the bone graft insertion to reinforce the void that was surgically created by the osteotomy and distraction process. Satisfactory results have been reported for the patients who underwent open wedge HTO with bone graft ${ }^{(23,38)}$. Biomechanical study evaluated that significant stress will be applied to the plate and lateral cortex of the osteotomy site under axial loading ${ }^{(15)}$ as the stress on the plate and the lateral cortex with using bone graft decreased significantly compared with no bone graft. Our study assumed two different scenarios regarding presence of bone graft. First, by creating a FE model without bone graft, the most unstable case was simulated as to represent a stage immediately after surgery when no bony fusion or bridging has occurred to offer load-sharing by the bone graft. On the other hand, with FE model with bone graft, full bony union between the bone graft and the host tibia was assumed to replicate a fully healed condition after surgery. Our results support the use of bone graft material at the osteotomy site as our FE results showed that the stress distribution at the lateral cortex bone and bone screw as well as structural rigidity.

Collapse of the lateral cortex is believed to be one of the most critical sources of instability at the osteotomy site which may lead to screw failure or loss of correction ${ }^{(17,22)}$. Recent clinical study reported that directing the plane of the osteotomy within the 'safe' zone cause is one way of preventing the lateral cortex bone fracture by keeping the osteotomy line away from the periosteal-lateral capsule of the tibiofibular joint during expansion of osteotomy gap. Indeed, our results confirmed that the fracture risk of the lateral cortex bone increased as the osteotomy level was moved further away from the periosteal-lateral capsule to the tibial plateau (from the 'lower' level to the 'upper' level as in our case presentation) regardless loading conditions. Especially, increase in the fracture risk under torsional loading was more evident as the moment arm from the center point of tibia plateau to osteotomy level increased with rise of osteotomy level and it was caused by loading points that are close to the ostetotomy level. However, magnitude of sensitivity of the changes in osteotomy level on the stress distribution and overall stability was relatively less significant as compared to the presence of bone graft or completion of bony union.

Our results demonstrated that fracture risk of the bone plate showed the opposite pattern from that of the lateral cortex. It was reduced as the osteotomy level was varied from the 'lower' level to the 'upper' level, perhaps due to the same reasons stated earlier with change in moment arm of the compression-bending load. Under torsional load, the risk remained relatively unchanged with regard to changes in osteotomy level.

Overall post-op structural rigidity was found to be primarily dependent on the presence 
of bone graft (or completion of bone healing process) whereas the osteotomy line was less significant factor. The trend was very similar to that of the lateral cortex results. Therefore, achieving full bony union with help of tri-cortical bone graft and osteotomy line ending near the tibiofibular joint (the 'lower' zone) would likely to assure the greatest post-operative stability.

Our overall FE results confirmed the clinical significance of the 'safe' zone. More precisely, the osteotomy line near the lower end of the 'safe' zone was found to be the most optimal case because it would least likely to cause fractures of the lateral cortex or the bone plate while assuring relative stability of the 'lower' zone. It would be very difficult to achieve the 'lower' zone orientation of the osteotomy line without compromising the tibiofibular joint or surrounding soft tissue. In fact, the presence of the soft tissue would provide additional support and reduce the chance of the lateral cortex disruption.

Regarding the limitations of this study, material properties of our tibia bone model may have been different from that of actual patient because the post-operative FE model was based on the tibia of a normal young individual. As decrease of bone mineral density can be found in most patients for open wedge HTO along with degeneration of the knee joint, more precise representation of the material properties of elderly patients simulating actual progression of osteoporosis would result in better understanding of the surgical factors involved ${ }^{(39)}$. However, our results were in good agreement with clinical findings. Further refining of FE models with more realistic representation of anatomical elements and clinical conditions of patients would be able to elucidate the sensitivities of surgical factors in HTO and offer more optimal surgical techniques.

\section{Conclusion}

Our FE assessment demonstrated that the fracture risk of lateral cortex bone and fixation plate as well as structural rigidity can be greatly influenced by various factors in surgery such as presence of bone graft, osteotomy line orientation, and loading pattern. The most critical factor was the presence of the bone graft, as the complete bony union between the bone graft and surrounding tibial tissues offered the highest structural rigidity and the least likelihood of lateral cortex fractures. The optimal osteotomy line was found to be near the 'lower' zone. However, the 'safe' zone offered relatively similar results to those of the 'lower' zone, and it was still superior to the 'upper' zone. Considering the surgical difficulties associated with achieving actual orientation of the 'lower' zone without inflicting damages to nearby tibiofibular joint, the osteotomy line near the lower end of the 'safe' zone was indeed the safest and most practical surgical approach as suggested in previous clinical studies. In conclusion, our results suggested the use of bone graft with safe level osteotomy to assure the greatest post-operative stability and to reduce the likelihood of complications such as the lateral cortex fractures and bone plate failure.

\section{References}

(1) Jackson R.W. and Abe I., The roll of arthroscopy in the management of disorders of the knee, JBJS, Vol. 54 (1972), pp. 310-322.

(2) Klinger H.M., Lorenz F. and Härer T., Open wedge tibial osteotomy by hemicallotasis for medial compartment osteoarthritis, Arch Orthop Trauma Surg, Vol. 121 (2001), pp. 245-247

(3) Franco V., Cerullo G., Cipolla M., Gianni E. and Puddu G., Open wedge high tibial osteotomy, Techniques in Knee Surgery, Vol. 1, No. 1 (2002), pp. 43-53.

(4) Brouwer R.W., Bierma-Zeinstra S.M., van Koeveringe A.J. and Verhaar J.A., Patellar height and the inclination of the tibial plateau after high tibial osteotomy: the open 
versus the closed-wedge technique, JBJS, Vol. 87, No. 9 (2005), pp. 1227-1232.

(5) Flecher X., Parratte S., Aubaniac J.M. and Argenson J.N., A 12-28-year follow-up study of closing wedge high tibial osteotomy, Clinical Orthopaedics \& Related Research, Vol. 452 (2006), pp. 91-96.

(6) Tunggal J.A., Higgins G.A. and Waddell J.P., Complications of closing wedge high tibial osteotomy, International Orthopaedics, Vol. 34, No. 2 (2010), pp. 255-261

(7) Matar W.Y., Boscariol R. and Dervin G.F., Open wedge high tibial osteotomy, The American Journal of Sports Medicine, Vol. 37, No. 4 (2009), pp. 735-742.

(8) Hernigou P. and Ma W., Open wedge tibial osteotomy with acrylic bone cement as bone substitute, The Knee, Vol. 8, No. 2 (2001), pp. 103-110.

(9) DeMeo P.J., Johnson E.M., Chiang P.P., Flamm A.M. and Miller M.C., Midterm follow-up of opening-wedge high tibial osteotomy, The American Journal of Sports Medicine, Vol.38, No. 10 (2010), pp. 2077-2084

(10) Hoell S., Suttmoeller J., Stoll V., Fuchs S. and Gosheger G., The high tibial osteotomy, open versus closed wedge_a comparison of methods in 108 patients, Arch Orthop Trauma Surg, Vol. 125 (2005), pp. 638-643

(11) Spahn G., Complications in high tibial (medial opening wedge) osteotomy, Archives of Orthopaedic and Trauma Surgery, Vol. 124, No. 10 (2004), pp. 649-653.

(12) Nelissen E.M., van Langelaan E.J. and Nelissen R.G., Stability of medial opening wedge high tibial osteotomy: a failure analysis, International Orthopaedics, Vol. 34 (2010), pp. 217-223.

(13) Agneskirchner J.D., Freiling D., Hurschler C. and Lobenhoffer P., Primary stability of four different implants for opening wedge high tibial osteotomy, Knee Surg Sports Traumatol Arthrosc, Vol. 14 (2006), pp. 291-300.

(14) Esenkaya I., Misirlioglu M., Kelestemur M.H., Elmali N. and Fadillioglu E., Biomechanical evaluation of different fixation plates in medial opening upper tibial osteotomy, The Knee, Vol. 14 (2007), pp. 46-50.

(15) Takeuchi R., Bito H., Akamatsu Y., Shiraishi T., Morishita S., Koshino T. and Saito T., In vitro stability of open wedge high tibial osteotomy with synthetic bone graft, The Knee, Vol. 17 (2010), pp. 217-220

(16) Blecha L.D., Zambelli P.Y., Ramaniraka N.A., Bourban P.E., Månson J.A. and Pioletti D.P., How plate positioning impacts the biomechanics of the open wedge tibial osteotomy; a finite element analysis, Comput Methods Biomech Biomed Engin, Vol. 8, No. 5 (2005), pp. 307-313

(17) Miller B.S., Dorsey W.O., Bryant C.R. and Austin J.C., The effect of lateral cortex disruption and repair on the stability of the medial opening wedge high tibial osteotomy, The American Journal of Sports Medicine, Vol. 33, No. 10 (2005), pp. $1552-1557$

(18) Rietbergen B., Weinans H., Huiskes R. and Odgaard A., A new method to determine trabecular bone elastic properties and loading using micromechanical finite-element models, J. Biomechanics, Vol. 28, No. 1 (1995), pp. 69-81.

(19) Taylor M., Tanner K.E. and Freeman M.A., Finite element analysis of the implanted proximal tibia: a relationship between the initial cancellous bone stresses and implant migration, J. Biomechanics, Vo. 31, No. 4 (1998), pp. 303-310.

(20) Completo A., Fonseca F. and Simões J.A., Finite element and experimental cortex strains of the intact and implanted tibia, J. Biomechanics Engineering, Vol. 129, No. 5 (2007), pp. 791-797

(21) Jacobi M., Wahl P. and Jakob R.P., Avoiding intraoperative complications in open-wedge high tibial valgus osteotomy: technical advancement, Knee Surg Sports Traumatol Arthrosc, Vol. 18 (2010), pp. 200-203

(22) Han S.B., Lee D.H., Shetty G.M., Chae D.J., Song J.G. and Nha K.W., A "safe zone" 
in medial open-wedge high tibia osteotomy to prevent lateral cortex fracture, Knee Surg Sports Traumatol Arthrosc, (2011), pp. 1-6

(23) Chae D.J., Shetty G.M., Lee D.B., Choi H.W., Han S.B. and Nha K.W., Tibial slope and patellar height after opening wedge high tibia osteotomy using autologous tricortical iliac bone graft, The Knee, Vol. 15 (2008), pp. 128-133

(24) Sgaglione N.A., Moynihan D.P. and Uggen C., The use of allografts in high tibial osteotomy: opening wedge technique, Oper Tech Sports Med, Vol. 15 (2007), pp. $82-80$

(25) Younger E.M. and Chapman M.W., Morbidity at bone graft donor sites, J Orthop Trauma, Vol.3 (1989), pp. 192-195

(26) Lim T.H., Eck J.C., An H.S., Hong J.H., Ahn J.Y. and You J.W., Biomechanics of transfixation in pedicle screw instrumentation, Spine, Vol. 21 (1996), pp. 2224-2229

(27) Cordey J., Borgeaud M. and Perren S.M., Force transfer between the plate and the bone: relative importance of the bending stiffness of the screws and the friction between plate and bone, Injury, Vol. 31 (2000), pp. 21-28

(28) Halloran J.P., Petrella A.J. and Rullkoetter P.J., Explicit finite element modeling of total knee replacement mechanics, J. Biomechanics, Vol. 38 (2005), pp. 323-331

(29) Lobenhoffer P., kniegelenknahe osteotomien, Georg Thieme Verlag (2007), pp. 1-161

(30) Paley D. and Pfeil J., Principles of deformity correction around the knee, Orthopedics, Vol. 29, No. 1 (2000), pp. 18-38.

(31) Sonoda N., Chosa E., Totoribe K. and Tajima N., Biomechanical analysis for stress fractures of the anterior middle third of the tibia in athletes: nonlinear analysis using a three-dimensional finite element method, J. orthopedic Science, Vol. 8 (2003), pp. 505-513

(32) Taylor D., O'Reilly P., Vallet L. and Lee T.C., The fatigue strength of compact bone in torsion, J. biomechanics, Vol. 36 (2003), pp. 1103-1109

(33) Zioupos P., Gresle M. and Winwood K., Fatigue strength of human cortical bone: Age, physical and material heterogeneity effects, J. Biomedical Materials Research Part A, Vol. 86A (2008), pp. 627-636

(34) Niinomi M., Mechanical properties of biomedical titanium alloys, Materials Science and Engineering: A, Vol. 243 (1998), pp. 231-236

(35) Stoffel K., Stachowiak G. and Kuster M., Open wedge high tibial osteotomy: biomechanical investigation of the modified Arthrex Osteotomy Plate (Puddu Plate) and the TomoFix Plate, Clinical Biomechanics, Vol. 19 (2004), pp. 944-950

(36) Zaki S.H. and Rae P.J., High tibial valgus osteotomy using the Tomofix plate: Medium-term results in young patients, Acta Orthopcedica Belgica, Vol. 75 (2009), pp. 360-367

(37) Staubli A.E., De Simoni C., Babst R. and Lobenhoffer P., TomoFix: a new LCP-concept for open wedge osteotomy of the medial proximal tibia--early results in 92 cases, Injury, Vol. 34 (2003), pp. 55-62

(38) Koshino T., Murase T. and Saito T., "Medial opening-wedge high tibial osteotomy with use of porous hydroxyapatite to treat medial compartment osteoarthritis of the knee, JBJS, Vol. 85 (2003), pp.78-85

(39) Li B. and Aspden R.M., Composition and mechanical properties of cancellous bone from the femoral head of patients with osteoporosis or osteoarthritis, J. Bone and Mineral Research, Vol. 12, No. 4 (1997), pp. 641-651 